\title{
Nodding syndrome may be an autoimmune reaction to the parasitic worm Onchocerca volvulus
}

2017 (c) The Authors, some rights reserved; exclusive licensee American Association for the Advancement of Science.

\author{
Tory P. Johnson, ${ }^{1}$ Richa Tyagi, ${ }^{1}$ Paul R. Lee, ${ }^{1}$ Myoung-Hwa Lee, ${ }^{1}$ Kory R. Johnson, ${ }^{2}$ \\ Jeffrey Kowalak, ${ }^{3}$ Abdel Elkahloun, ${ }^{4}$ Marie Medynets, ${ }^{5}$ Alina Hategan, ${ }^{1}$ Joseph Kubofcik, $^{6}$ \\ James Sejvar, ${ }^{7}$ Jeffrey Ratto, ${ }^{8}$ Sudhir Bunga, ${ }^{8}$ Issa Makumbi, ${ }^{9}$ Jane R. Aceng, ${ }^{9}$ Thomas B. Nutman, ${ }^{6}$ \\ Scott F. Dowell, ${ }^{10}$ Avindra Nath ${ }^{1 *}$
}

\begin{abstract}
Nodding syndrome is an epileptic disorder of unknown etiology that occurs in children in East Africa. There is an epidemiological association with Onchocerca volvulus, the parasitic worm that causes onchocerciasis (river blindness), but there is limited evidence that the parasite itself is neuroinvasive. We hypothesized that nodding syndrome may be an autoimmune-mediated disease. Using protein chip methodology, we detected autoantibodies to leiomodin-1 more abundantly in patients with nodding syndrome compared to unaffected controls from the same village. Leiomodin-1 autoantibodies were found in both the sera and cerebrospinal fluid of patients with nodding syndrome. Leiomodin-1 was found to be expressed in mature and developing human neurons in vitro and was localized in mouse brain to the CA3 region of the hippocampus, Purkinje cells in the cerebellum, and cortical neurons, structures that also appear to be affected in patients with nodding syndrome. Antibodies targeting leiomodin-1 were neurotoxic in vitro, and leiomodin-1 antibodies purified from patients with nodding syndrome were cross-reactive with $O$. volvulus antigens. This study provides initial evidence supporting the hypothesis that nodding syndrome is an autoimmune epileptic disorder caused by molecular mimicry with $O$. volvulus antigens and suggests that patients may benefit from immunomodulatory therapies.
\end{abstract}

\section{INTRODUCTION}

Nodding syndrome is an epileptic disorder typified by atonic seizures that affects children between 5 and 15 years of age in geographically localized regions of Tanzania, Uganda, and the Republic of South Sudan (1). The atonic seizures are characterized by a head-dropping motion, but patients may also develop clonic-tonic seizures, atypical absence seizures, mild to severe cognitive impairment, and cerebellar, cerebral, and hippocampal atrophy (1-5). Nodding syndrome is a disabling disease, resulting in neurological deterioration and, in some cases, death $(1,2)$. The clinical characteristics of nodding syndrome are distinct from other epileptic disorders in children.

Because of an increase in reports of nodding syndrome (6), rigorous efforts to understand this disease have been undertaken (2). These studies have resulted in a consensus case definition and clinical characterization of nodding syndrome (1-4). However, the pathophysiology and etiology of nodding syndrome remain unknown. Extensive investigation of environmental neurotoxins, nutritional deficiencies, genetic disorders, or infectious organisms has been unrevealing (2). An increased rate of

\footnotetext{
${ }^{1}$ Section of Infections of the Nervous System, National Institute of Neurological Disorders and Stroke, National Institutes of Health, Bethesda, MD 20892, USA. ${ }^{2}$ Bioinformatics Section, National Institute of Neurological Disorders and Stroke, National Institutes of Health, Bethesda, MD 20892, USA. ${ }^{3}$ Clinical Proteomics Unit, National Institute of Neurological Disorders and Stroke, National Institutes of Health, Bethesda, MD 20892, USA. ${ }^{4}$ Microarray Core Facility, National Human Genome Research Institute, National Institutes of Health, Bethesda, MD 20892, USA. ${ }^{5}$ Neural Differentiation Unit, National Institute of Neurological Disorders and Stroke, National Institutes of Health, Bethesda, MD 20892, USA. ${ }^{6}$ Helminth Immunology Section, Laboratory of Parasitic Diseases, National Institute of Allergy and Infectious Diseases, National Institutes of Health, Bethesda, MD 20892, USA. 'Division of High-Consequence Pathogens and Pathology, National Center for Emerging and Zoonotic Infectious Diseases, Centers for Disease Control and Prevention, Atlanta, GA 30333, USA. ${ }^{8}$ Division of Global Health Protection, Center for Global Health, Centers for Disease Control and Prevention, Atlanta, GA 30333, USA. ${ }^{9}$ Ministry of Health, Kampala, Uganda. ${ }^{10}$ Bill \& Melinda Gates Foundation, Seattle, WA 98109, USA.

${ }^{*}$ Corresponding author. Email: natha@ninds.nih.gov
}

nodding syndrome in areas where the parasite Onchocerca volvulus is endemic led to the hypothesis that the infection may play a role in nodding syndrome pathogenesis (6). Case-control studies have consistently documented an association between nodding syndrome and $O$. volvulus infection but have failed to find evidence of invasion of the brain or cerebrospinal fluid (CSF) by the mature parasite $(2,5,7)$, although prelarval worms (microfilariae) have been detected in the CSF (8). It has thus been hypothesized that an immune-mediated mechanism may be involved. Previous investigations of autoantibodies known to be associated with neurological illness have been unrevealing in nodding syndrome [as described in $(2,9)]$. The aim of the current study was to further investigate whether autoantibodies could be a contributing factor to the pathogenesis of nodding syndrome.

\section{RESULTS}

\section{Autoantibodies in patients with nodding syndrome}

An unbiased approach for profiling autoantibodies using a protein array detected a $>2$-fold increase in reactivity to 167 probes representing 137 individual proteins and a $>100$-fold increase in four proteins in pooled sera from patients with nodding syndrome compared to pooled sera from unaffected control villagers (Fig. 1A and table S1). The top two signals were from autoantibodies to leiomodin-1 (increased 33,000-fold) and autoantibodies to DJ-1 (increased 750-fold). Further examination of the top four enriched autoantibodies in patients with nodding syndrome (table S2) demonstrated differential immunoreactivity by immunoblot analyses between pooled serum samples from patients with nodding syndrome and controls for only two of the proteins, leiomodin-1 and DJ-1 (Fig. 1B). However, only antibodies to leiomodin-1 (and not to DJ-1) were detected in the CSF of patients with nodding syndrome (Fig. 1C). Serum samples from each of the patients with nodding syndrome and unaffected village controls were analyzed for reactivity to leiomodin-1 by enzyme-linked immunosorbent assay (ELISA) (Fig. 1D and Table 1); a subset of samples was 


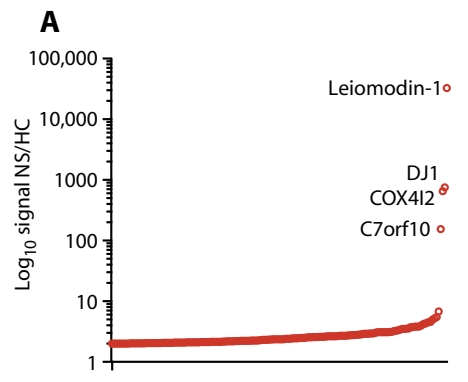

B
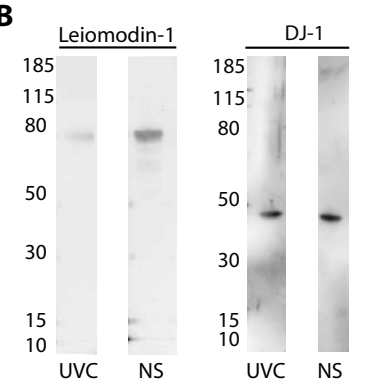

C

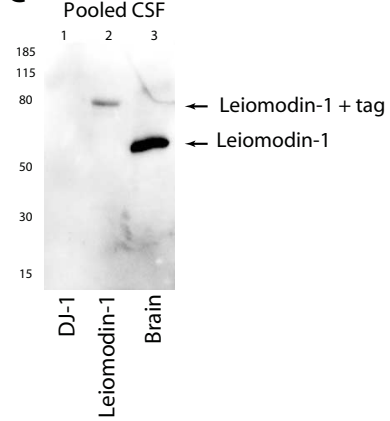

E

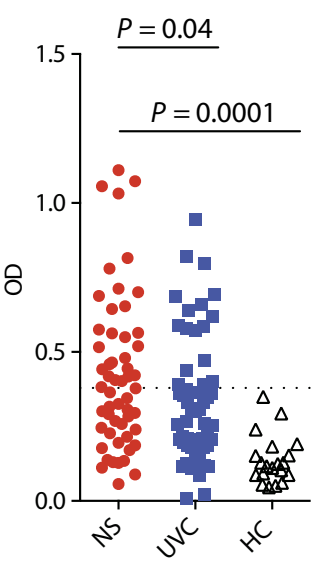

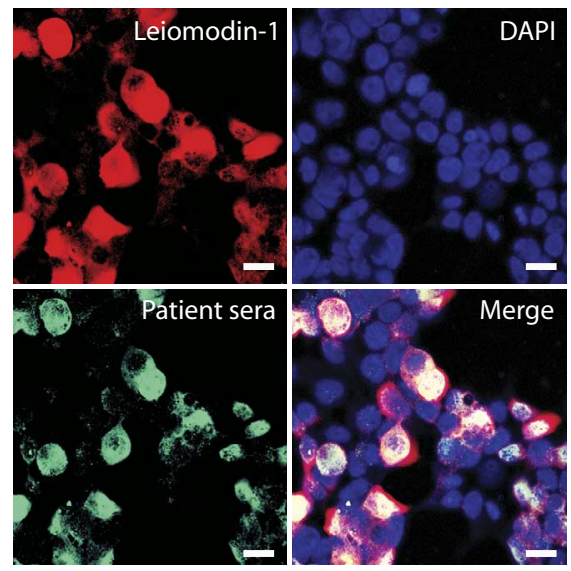

Fig. 1. Leiomodin-1 autoantibodies in patients with nodding syndrome. (A) $\log _{10}$-fold distribution plot depicting autoantibody reactivity differences between patients with nodding syndrome (NS) and unaffected village controls (UVC). Annotated on the graph are four proteins observed to have a $>100$-fold difference between nodding syndrome and unaffected village controls. (B) Immunoblot analyses of leiomodin-1 and DJ-1 immunoreactivity in sera from unaffected village controls or nodding syndrome patients. (C) Immunoblot analysis of recombinant DJ-1, recombinant leiomodin-1, and human brain homogenate probed with CSF pooled from 16 patients with nodding syndrome. In lane 1, there is no immunoreactivity to recombinant DJ-1. In lane 2, there is immunoreactivity to histidine-tagged leiomodin-1 (arrow; $\sim 80 \mathrm{kDa}$ ). Lane 3 shows immunoreactivity to a single protein in brain homogenate at $\sim 60 \mathrm{kDa}$, the molecular mass of leiomodin-1 (arrow). (D) Scatterplot depicting optical density (OD) of individual patient serum's immunoreactivity to leiomodin-1 as determined by ELISA. The cutoff for determining a positive sample was set at 3 SD above the mean for U.S. healthy control sera (HC; $n=20$ ). Data were analyzed by analysis of variance (ANOVA) that showed an overall significant difference $(P=0.0001)$, and a Sidak-Holms correction was applied to correct for multiple comparisons: nodding syndrome $(n=55)$ versus unaffected village controls $(n=55), P=0.04$. Data were also log-transformed and reanalyzed with consistent findings. (E) Coimmunostaining with patient sera (green) and rabbit anti-leiomodin-1 antibody (red). Nuclei were stained with 4',6-diamidino-2-phenylindole (DAPI) (blue). Scale bars, $20 \mu \mathrm{m}$.

negative cases. The same did not hold true for unaffected village controls. Additionally, patients with nodding syndrome had increased titers of antibodies to leiomodin-1 compared to unaffected village controls $(P=0.04$, ANOVA with Holm-Sidak correction for multiple comparisons). Both immunoglobulin G (IgG) and IgM antibodies directed against leiomodin-1 were present in the sera of patients with nodding syndrome (fig. S2). Fifty percent (8 of 16) of patients with nodding syndrome showed antibodies to leiomodin-1 in the CSF, whereas none ( 0 of 8 ) of the North American patients with epilepsy, as a control, demonstrated antibodies to leiomodin-1 in their $\operatorname{CSF}(P=0.022$, Fisher's exact test).

To confirm that antibodies in the sera from patients with nodding syndrome recognized leiomodin-1, human embryonic kidney (HEK) cells transfected with leiomodin-1 DNA were co-stained with rabbit anti-leiomodin-1 antibody and sera from a patient with leiomodin-1 antibodies or sera from an unaffected village control without antibodies to leiomodin-1. Colocalization of nodding syndrome patient sera, but not the sera from unaffected village controls, with leiomodin-1 was observed (Fig. 1E and fig. S3). To confirm that the antibodies in the CSF from patients recognized neuronal leiomodin-1, we stained human neurons with CSF from two patients with leiomodin-1 antibodies in conunction with rabbit anti-leiomodin-1. Colocalization of the human antibodies from CSF and the rabbit antileiomodin-1 antibodies was observed on neurons (fig. S4). confirmed by immunoprecipitation (fig. S1). Leiomodin-1 antibodies were more frequently detected in patients with nodding syndrome compared to unaffected village controls: 29 of 55 (52.7\%) patients with nodding syndrome versus 17 of $55(30.9 \%)$ unaffected village controls $[P=0.024$, mOR, $2.7 ; 95 \%$ confidence interval (CI), 1.1 to 6.5$]$. In patients with nodding syndrome with determined $O$. volvulus status ( $n=54), 44$ patients were $O$. volvulus-positive. Of these, 24 patients (54.5\%) were positive for both $O$. volvulus and leiomodin-1 antibodies. Twenty patients (45.5\%) were O. volvulus-positive and leiomodin-1 antibodynegative. In unaffected village controls, there were 54 individuals with determined $O$. volvulus status. Of these controls, 29 were $O$. volvulus-positive. Twelve of these 29 controls (41.4\%) were leiomodin-1 antibody-positive and $O$. volvulus-positive, and $58.6 \%$ were leiomodin- 1 antibodynegative and $O$. volvulus-positive. Thus, in patients with nodding syndrome, there was a higher percentage of $O$. volvulus-positive status in leiomodin-1 antibody-positive cases than in leiomodin-1 antibody-

\section{Expression of leiomodin-1 in the CNS}

Because leiomodin-1 has been reported to be expressed primarily in smooth muscle tissue and in the thyroid $(10,11)$, we next examined the expression of leiomodin-1 in the central nervous system (CNS). Using multiple approaches, we confirmed that leiomodin-1 is expressed in the CNS and in neurons. Immunostaining of human neurons with rabbit anti-leiomodin-1 antibody demonstrated leiomodin-1 expression throughout the cytoplasm of neurons (Fig. 2A). Immunoblots demonstrated the presence of leiomodin-1 in adult human brain homogenates (Fig. 2B). Localization of leiomodin-1 in mouse brain by immunohistochemistry using rabbit anti-leiomodin-1 antibody showed that it was focally expressed in cortical neurons in the cerebral cortex, Purkinje cells in the cerebellum, and pyramidal cells in the CA3 region of the hippocampus. Other regions of the hippocampus including CA1 and the dentate gyrus did not express leiomodin-1 (Fig. 2C). Smooth muscle from blood vessels and skeletal muscle (Fig. 2D) were used as 
Table 1. Patient demographics, Onchocerca infection status, and presence of leiomodin-1 antibodies. mOR, matched odds ratio; NS, not significant.

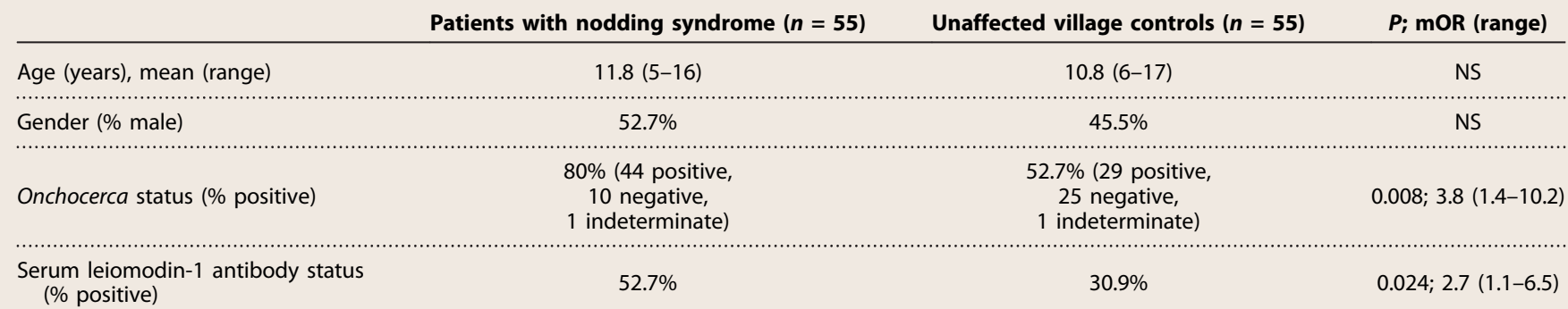

positive controls. Mining publicly available RNA sequencing data, we confirmed that leiomodin-1 is expressed in the human brain (fig. S5). This was confirmed by detecting leiomodin-1 messenger RNA (mRNA) in human brain tissue extracts and human neuronal stem cells and cultured neurons (Fig. 2E and table S3). There was a higher expression of leiomodin$1 \mathrm{mRNA}$ in brain extracts compared to the expression in human neural stem cells and differentiated neurons.

\section{Leiomodin-1 antibodies are neurotoxic}

To determine whether antibodies to leiomodin-1 could cause neuronal dysfunction, we treated cultured human neurons with leiomodin- 1 antibodies or control serum and assayed them for viability. Neurons treated with anti-leiomodin-1 antibody showed decreased viability (mean viability $\pm \mathrm{SD}, 40.9 \pm 9.3 \%$ ) compared to treatment with normal rabbit sera (mean viability $\pm \mathrm{SD}, 75.5 \pm 12.8 \% ; P=3.4 \times 10^{-9}$, two-way Student's paired $t$ test) (Fig. 3A and table S4). Neurons treated with sera from a patient with nodding syndrome (1:200) with detectable leiomodin1 antibodies demonstrated neurotoxicity (mean viability \pm SD, $14.7 \pm$ $1.0 \%$ ) that was abrogated by antibody depletion from the patient sera (mean viability $\pm \mathrm{SD}, 78 \pm 12.8 \% ; P=0.0003$, two-way Student's paired $t$ test) (Fig. $3 \mathrm{~B}$ and table S5). Neurons exposed to sera from patients with detectable leiomodin-1 antibodies $(n=4)$ showed increased neurotoxicity (mean toxicity \pm SD, $52.71 \pm 24.9 \%$ ) compared to sera selectively depleted of leiomodin-1 antibodies by affinity purification (mean toxicity $\pm \mathrm{SD}, 33.16 \pm 19.8 \% ; P=0.0048$, two-way Student's paired $t$ test) (Fig. 3C and table S6). The neurotoxicity induced by autoantibodies to leiomodin-1 from patients with nodding syndrome in conjunction with the expression of leiomodin-1 in the CNS suggests that antibodies directed against leiomodin-1 may play a role in nodding syndrome disease pathogenesis.

\section{Leiomodin-1 antibodies cross-react with O. volvulus proteins}

Epidemiological studies have indicated that nodding syndrome is associated with $O$. volvulus $(2,4-6,12-14)$. Therefore, we investigated whether autoantibodies to leiomodin- 1 could be related to infection with $O$. volvulus. Screening of antigen prepared from adult male and female $O$. volvulus with pooled sera from patients with nodding syndrome and from unaffected village controls by immunoblotting showed limited differential immunoreactivity profiles (Fig. 4A). Bands to which nodding syndrome patient sera demonstrated increased immunoreactivity were excised from the gel and identified by mass spectrometry (table S7). O. volvulus tropomyosin was one of the proteins identified. O. volvulus tropomyosin protein has $25.5 \%$ sequence identity and $37 \%$ sequence similarity to leiomodin-1 (Fig. 4B). Although the overall homology is low, regional homology analyses indicated that the conserved N-terminal DAIKK sequence of $O$. volvulus tropomyosin (15), amino acids 2 to 14 , showed $57.1 \%$ identity and $71.4 \%$ similarity to amino acids 107 to 117 of human leiomodin-1. The conserved signature sequence LKEAExRAE (15), amino acids 230 to 240 of $O$. volvulus tropomyosin, showed $66.7 \%$ identity and $83.3 \%$ similarity to amino acids 362 to 372 of human leiomodin-1. Although many of the proteins from $O$. volvulus remain uncharacterized, we hypothesized that there may be homology between human leiomodin-1, a member of the tropomodulin gene family (10), and tropomodulin from $O$. volvulus. Modeling of human leiomodin-1 and $O$. volvulus tropomodulin demonstrated regions of structural overlap (Fig. 4C, left). Further modeling showed that the second hit in our screen, human DJ-1, also had strong homology to the O. volvulus homolog (Fig. 4C, right). This in silico analysis suggested that infection with $O$. volvulus may generate antibodies crossreactive to host proteins. We therefore aimed to determine whether autoantibodies to leiomodin-1 cross-reacted with $O$. volvulus proteins.

Affinity-purified leiomodin-1 antibodies from four patients with nodding syndrome showed a single band after addition of lysates from HEK cells expressing leiomodin-1, indicating that these antibodies were specific for human leiomodin-1 (Fig. 4D). However, these antibodies also robustly recognized several proteins in an $O$. volvulus whole-organism lysate, suggesting that several $O$. volvulus proteins may share homology with human leiomodin-1 (Fig. 4D). Further confirmation of the cross-reactive nature of these antibodies was demonstrated by competing for the binding of leiomodin- 1 by nodding syndrome patient sera $(n=4)$ after preincubation of patient sera with either $O$. volvulus whole-organism lysate or a nonspecific protein, BSA. Sera that were preincubated with $O$. volvulus lysate showed decreased immunoreactivity to leiomodin-1 (mean OD, $78.3 \pm$ 89.3) compared to sera preincubated with BSA (mean OD, $236.7 \pm 124 ; P=$ 0.042 , one-way Student's unpaired $t$ test) (Fig. $4 \mathrm{E}$ and table $\mathrm{S} 8$ ).

\section{DISCUSSION}

Nodding syndrome is a devastating disease for children, families, and communities. A 2012-2013 survey estimated that there were about 2000 cases in Northern Uganda alone $(2,16)$. Despite international efforts to identify the etiological factors, the pathophysiology of nodding syndrome remains elusive, and no cure is available (12). Here, we hypothesized that an autoimmune process contributes to nodding syndrome. To address this hypothesis, we compared affected patients and unaffected village controls by conducting detailed immunotyping for autoreactive antibodies. Our initial attempt to discover autoantibodies by 

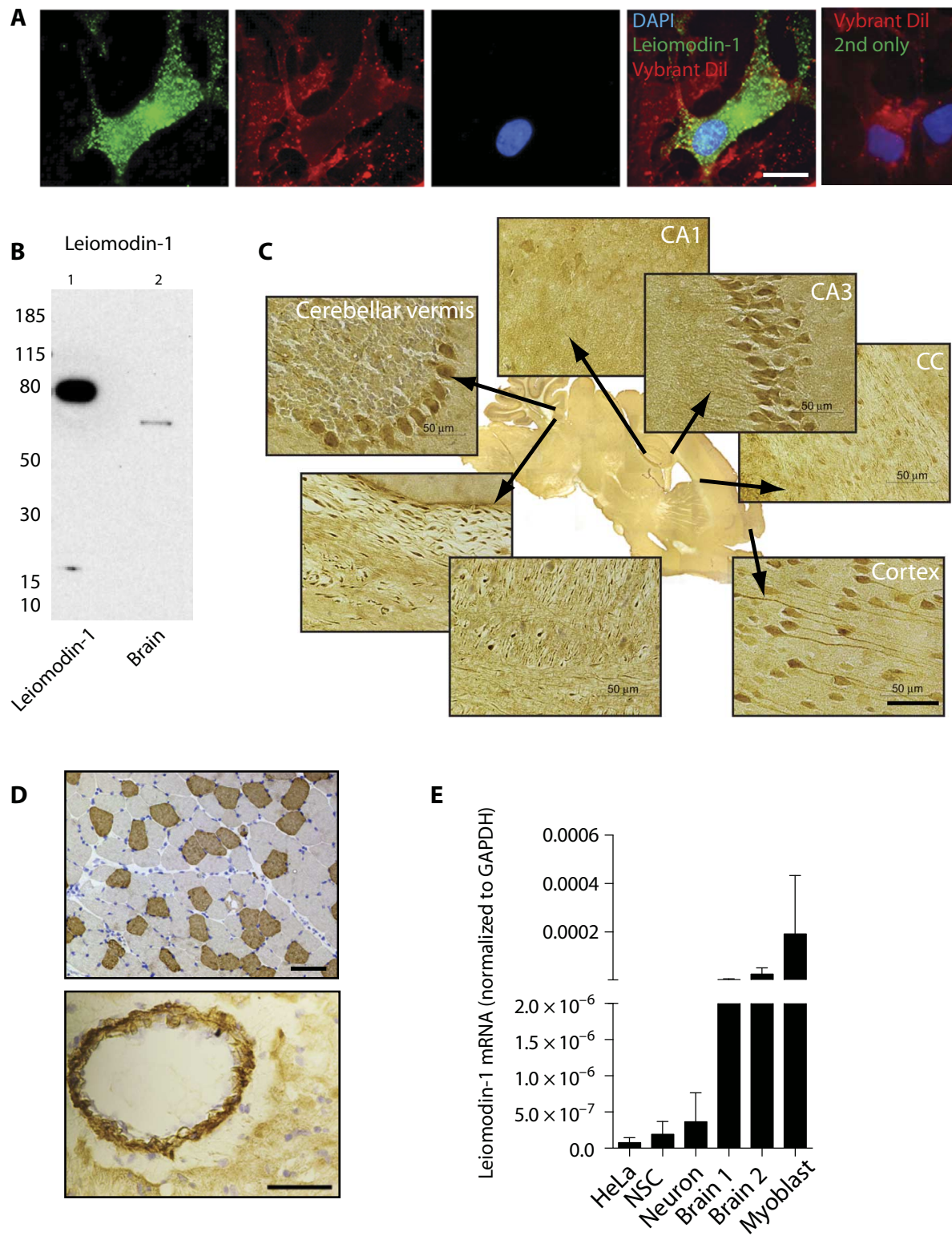

Fig. 2. Leiomodin-1 is expressed in human brain. (A) Cultured human neurons immunostained with rabbit antileiomodin-1 antibody (green channel). Vybrant Dil labels the cell membrane (red), and DAPI (blue) indicates the nucleus. Vybrant Dil and secondary antibody (2nd) only (rightmost). Scale bars, $20 \mu \mathrm{m}$. (B) Immunoblot analysis of human brain homogenates with rabbit anti-leiomodin-1 antibody and recombinant leiomodin-1 plus histidinetag as a positive control. (C) Immunohistochemistry demonstrates leiomodin-1 staining in specific areas of the murine brain. Scale bar, $50 \mu \mathrm{m}$. (D) Murine skeletal muscle (top; scale bar, $100 \mu \mathrm{m}$ ) and murine smooth muscle in the wall of a blood vessel (bottom; scale bar, $50 \mu \mathrm{m}$ ) as positive controls. (E) Quantitative polymerase chain reaction (qPCR) measuring leiomodin-1 transcripts in HeLa cells (as a low-expressing positive control), human neuronal stem cells (NSCs), human neurons, brain homogenates from two individuals (brain 1 and brain 2), and H9C2 myoblast cells (as a high-expressing positive control). Data are expressed as the mean leiomodin-1 transcript levels [normalized to glyceraldehyde-3phosphate dehydrogenase $(\mathrm{GAPDH})] \pm$ SD from three independent replicates. (Tabulated data are available in the Supplementary Materials.)

using a protein chip showed the presence of antibodies to multiple antigens. Four of these had levels greater than 100 -fold higher in patients compared to the unaffected village controls, demarcating them from the other antibodies that showed less than a 10-fold increase. Of the four proteins, autoantibodies to only two, DJ-1 and leiomodin-1, were confirmed biochemically to have increased reactivity in patients compared

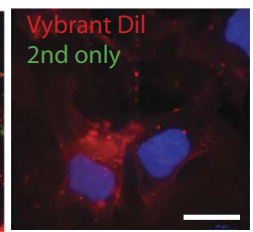

to controls. Although we were able to confirm the presence of antibodies to DJ-1 in sera from patients with nodding syndrome, it was not detectable in CSF. In contrast, antibodies to leiomodin-1 were present in the sera and CSF of about half the patients with nodding syndrome. Heretofore, leiomodin-1 was known to be present primarily in muscle $(10,11)$, but its location in the brain had not been characterized. Further analyses in our study showed that leiomodin-1 transcripts and protein were detectable in the CNS.

The immune response to leiomodin- 1 is of particular interest because we demonstrated that leiomodin-1 is expressed in neurons in distinct regions of the mouse brain, the cerebral cortex, the CA3 region of the hippocampus, and Purkinje cells in the cerebellum, areas that correspond with those hypothesized to be associated with the clinical manifestations of nodding syndrome. For example, patients with nodding syndrome have cerebral atrophy and associated cognitive abnormalities $(1,3,4,17)$. In animal models of epilepsy, retraction of dendrites and loss of neurons in the CA3 region are associated with epileptogenesis (18). This is also consistent with hippocampal and cerebellar atrophy seen upon magnetic resonance imaging of some patients with nodding syndrome $(1,3-5)$. Leiomodin-1, previously described as human $64-\mathrm{kDa}$ autoantigen D1 (19), acts as an actin nucleator in muscle cells (20). Except for the description of cold extremities (3), patients with nodding syndrome are not known to develop vascular symptoms. The specificity of CNS involvement may indicate that leiomodin-1 is differentially expressed, modified, or regulated in the CNS compared to other tissue compartments.

In contrast to other autoimmune epilepsies, where the antibodies are targeted against surface receptors such as AMPAR (AMPA-selective glutamate receptor), NMDAR ( $N$-methyl-D-aspartate receptor), AMPAR (AMPA-selective glutamate receptors), GABAR ( $\gamma$-aminobutyric acid receptor), VGKC (voltage-gated potassium channel), and the glycine receptor (21), leiomodin-1 is an intracellular antigen. Other intracellular antigens that have been associated with encephalitis and epilepsies include $\mathrm{Hu}, \mathrm{CRMP}-5$ (collapsin response mediator protein 5), GAD-65 (glutamic acid decarboxylase 65), and Ma (21). It may be that autoreactive $\mathrm{T}$ cells are the main mediators of the immunopathology of nodding syndrome with the production of leiomodin1 autoantibodies as a consequence of this T cell activation. Alternatively, 

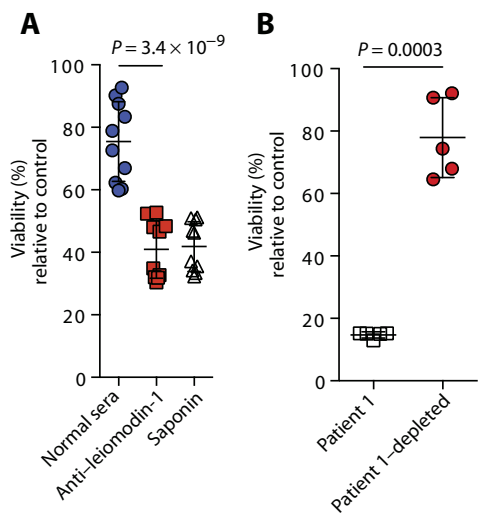

C

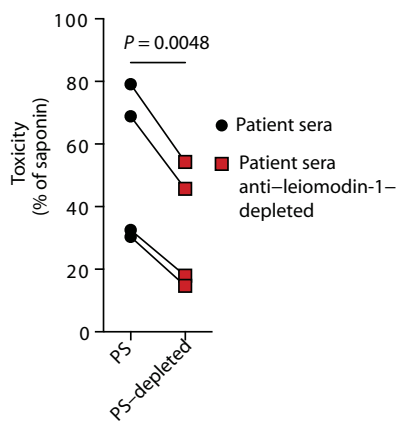

Fig. 3. Leiomodin-1 antibodies are neurotoxic. (A) Viability of primary human neurons treated with rabbit polyclonal anti-leiomodin-1 antibody compared to normal rabbit sera. Saponin was used as a positive neurotoxic control. Data shown are percent viability relative to treatment with vehicle only $\left(P=3.4 \times 10^{-9}\right.$; analyzed by repeatedmeasures ANOVA with Scheffe's procedure), with the horizontal bars indicating the mean viability \pm SD for $n=10$ replicates. (B) Viability of neurons treated with sera from patients with nodding syndrome with detectable anti-leiomodin-1 antibodies compared to antibody-depleted sera from the same patient $(P=0.0003$, two-way Student's paired $t$ test). Horizontal bars are the mean percent viability $\pm S D$ relative to cells in culture medium only of $n=5$ replicates. (C) Neurotoxicity induced by each patient's sera (PS) was compared to sera specifically depleted of leiomodin-1 antibodies (PS-depleted). Data shown are percent toxicity relative to saponin $(n=4 ; P=0.0048$, two-way Student's paired $t$ test). [Tabulated data are available for $(A)$ to $(C)$ in the Supplementary Materials.]

antibodies to leiomodin-1 may be directly contributing to disease. Although antibodies to intracellular antigens are not classically thought to be pathogenic, antibodies to intracellular neuronal targets such as amphiphysin have been shown to cause stiff person syndrome in an animal model (22). While the molecular mechanisms for antibodies that target intracellular antigens are not completely elucidated, some studies suggest cell-penetrating antibodies (22), alterations in antigen localization during apoptosis $(23,24)$, and changes in antigen expression within the target tissue during repair of injury (25). Additional studies of leiomodin-1 expression in the CNS, especially confirming subcellular localization during damage and repair processes, will be important.

Epidemiological studies have consistently shown an association between nodding syndrome and infection with $O$. volvulus $(2,4-6)$. We found that leiomodin-1 antibodies cross-reacted robustly with $O$. volvulus antigens and found sequence and structural homology between leiomodin-1 and O. volvulus tropomyosin and tropomodulin. Not all $O$. volvulus proteins have been fully characterized, and other parasite proteins could share homology. Further, our second hit, DJ-1, had structural homology to its $O$. volvulus homolog. We speculate that the typical immune response to $O$. volvulus infection may be generating multiple cross-reactive antibodies and that, in some patients, autoantibodies develop, enter the CNS, and cause distinct pathology recognized as nodding syndrome. However, not all patients with nodding syndrome had detectable antibodies to leiomodin-1. We speculate that nodding syndrome may not be a single antibody syndrome, and other investigations have suggested that patients with nodding syndrome may have antibodies to other neuronal proteins (26). The patients we investigated had multiple autoantibodies, as demonstrated by proteomics. Here, we have characterized the autoantibody with the highest titer that best distinguished the patients with nodding syndrome from unaffected village controls. This syndrome is likely not a disease mediated by a single immune specificity.
We postulate that the age of onset of nodding syndrome may be related to the initial burden of exposure to $O$. volvulus in early childhood. However, it is not clear why the syndrome is not seen in adults. It is possible that the developing immune system, or ongoing brain development, may make children more vulnerable to nodding syndrome. Additionally, some patients also have delayed sexual development, suggesting pituitary-hypothalamic dysfunction (27). In the current study, we have not investigated whether leiomodin-1 is expressed in these regions of the brain. The geographical restriction of nodding syndrome relative to the presence of $O$. volvulus may be due to interruption of parasite control in these regions (28) or may suggest that other as yet unidentified cofactors may also contribute to the pathobiology of nodding syndrome.

This study has several limitations that future studies need to address. We examined only a limited number of well-defined patients and unaffected village controls collected in the public health responses in Uganda and South Sudan in this cross-sectional discovery study. Although we found a statistically significant association between leiomodin-1 antibodies and nodding syndrome, one-third of unaffected village controls also had detectable antibodies. It may be that these individuals had early, asymptomatic disease that would progress to recognizable nodding syndrome. However, in this cross-sectional study, it was not possible to determine whether leiomodin-1 serum antibodies were an early marker of disease. Future studies, especially longitudinal studies examining leiomodin-1 antibody titers, are needed, but because of the nature of the epidemic and the remote location, such studies will be challenging. It is possible that other pathophysiological changes in patients with nodding syndrome allow for penetration of leiomodin-1 antibodies into the CNS. However, at the time of sample collection, it was not appropriate to collect CSF from village children not displaying neurological disease. Future studies may be able to determine whether differences in antibody status in the CNS correlate with disease. Additionally, using a limited number of patients with antibodies to leiomodin-1, we demonstrated that these antibodies were neurotoxic. Although we did not investigate the toxicity of serum samples from unaffected village controls or patients with nodding syndrome who lacked detectable leiomodin-1 antibodies and hence could not exclude the possibility of other neurotoxic substances in these patients, we think that this is unlikely because the depletion of leiomodin- 1 antibodies decreased the neurotoxicity. Another limitation of this study was that only sera and CSF were investigated, and therefore, potentially important immune cells such as leiomodin-1-specific T cells could not be characterized. Autoreactive T cells may be the mediators of nodding syndrome with the production of leiomodin-1 autoantibodies as a result of this immune response. Further studies investigating the $\mathrm{T}$ cell repertoire of patients in both the periphery and CNS would be informative. Despite these limitations, the work reported here provides insight into the pathophysiological mechanisms that contribute to the development of nodding syndrome. This syndrome can now be added to a growing list of autoimmune epilepsies $(29,30)$.

One mechanism through which autoreactive immune components may be generated is molecular mimicry. Four criteria have been established to define a molecular mimicry process, including (i) evidence of an epidemiological association between the pathogen and the immune-mediated disease, (ii) demonstration of immune cells or antibodies directed against patient tissue containing the antigen, (iii) demonstration of cross-reactivity of immune cells or antibodies specific to the host antigen with the pathogen, and (iv) immune response to and reproduction of disease in an animal model by infection or immunization (31). Here, we have demonstrated (i) the presence of 


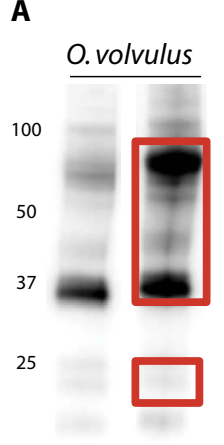

UVC NS
B

LMOD1_HUMAN
TPM_ONCVO
LMOD1_HUMAN
TPM_ONCVO
LMOD1_HUMAN
TPM_ONCVO
LMOD1_HUMAN
TPM_ONCVO
LMOD1_HUMAN
TPM_ONCVO
LMOD1_HUMAN
TPM_ONCVO
LMOD1_HUMAN
TPM_ONCVO

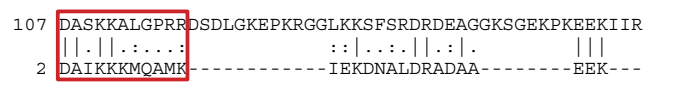

157 GIDKGRVRAAVDKKEAGKDGRGEERAVATKKEEEKKGSD - - - - - - - - - R

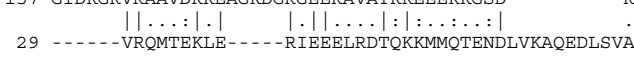

197 NTGLSRDKDKKREEMK-EVAKKEDDEKVKGERRNTDTRKEGEKMKRAGGN 68 NTNL-EDKEKKVQEAEAEVAAL-- - - - - - NRRMTLLEEE-

246 TDMKKEDEKVKRGTGN--- - TDTKKDDEKVKKNEPLHEKEAKDDSKTKTP

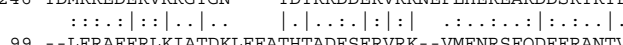
99 - LERAEERLKIATDKLEEATHTADESERVRK--VMENRSFQDEERANT 292 EKQTPSGPTKPSEGPAKVEEEAAP-SIFDEPLERVKN - - - NDPEMTEV-

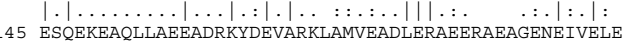
336 - - - - - NVNNSDCITNEILVR- - FTEALEFNTVVY LFALANTRADPH

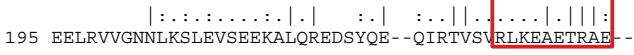
375 VAFAIAIMLKANKTITSLNLDSNH 398 ||$\ldots:|\ldots| \ldots \ldots|\ldots \ldots|$
241 - -FAERSVQKLQKKVDRLEDELVH

D

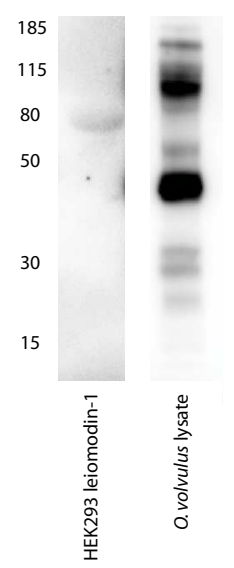

E

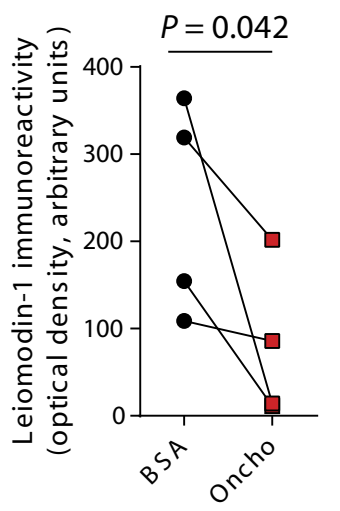

C

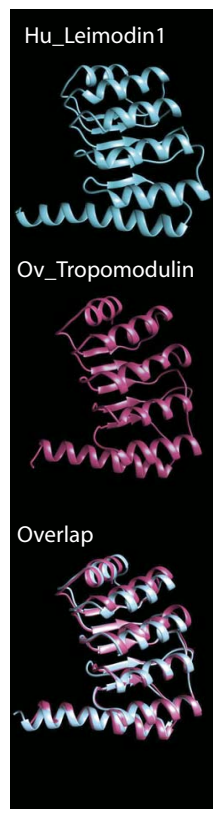

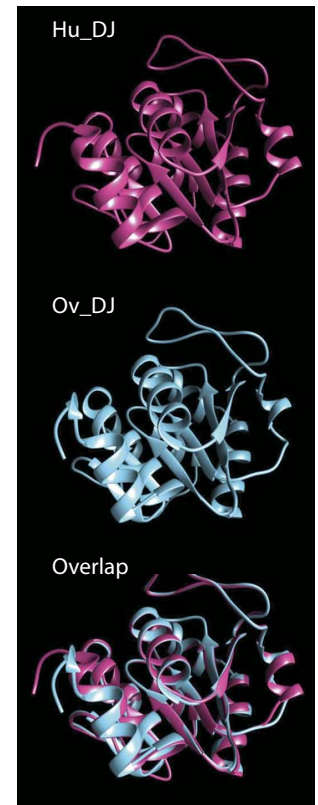

Fig. 4. Leiomodin-1 autoantibodies from the CSF of patients with nodding syndrome cross-react with $\mathbf{O}$. volvulus proteins. (A) Western blot analyses of $O$. volvulus lysates probed with sera from unaffected village controls and patients with nodding syndrome. Red boxes indicate the region of the corresponding gel that was excised and analyzed by mass spectrometry for protein identification. (B) Sequence homology between human leiomodin-1 (accession number NP_036266) and $O$. volvulus tropomyosin (accession number Q25632) by pairwise alignment (EMBOSS, EMBL EBI with Smith-Waterman algorithm). Conserved tropomyosin regions 1 and 2 and their corresponding leiomodin-1 sequences are indicated by red boxes. (C) Depiction of structural homology between human leiomodin-1 (Hu_Leiomodin1) and O. volvulus tropomodulin (Ov_Tropomodulin) (accession number A0A044S1K6) (left) and between human DJ1 (Hu_DJ) (accession number Q99497) and the O. volvulus ortholog (Ov_DJ) (accession number A0A044RAJ4) (right). (D) Leiomodin-1 antibodies purified from patients reacted to a single band in HEK293 cells overexpressing tagged human leiomodin-1 (left). The same purified anti-leiomodin-1 antibodies demonstrated strong immunoreactivity to multiple proteins from $O$. volvulus whole-organism lysate (right). (E) Inhibition of patient sera immunoreactivity to leiomodin-1 by $O$. volvulus whole-organism lysate (oncho) compared to bovine serum albumin (BSA) ( $n=4 ; P=0.042$, one-way Student's unpaired $t$ test). (Tabulated data are available in the Supplementary Materials.)

leiomodin-1 antibodies with titers substantially higher in patients than in controls; (ii) the presence of leiomodin-1 antibodies in the CSF of patients with nodding syndrome; (iii) homology between leiomodin-1 and $O$. volvulus proteins, both with sequence data and biochemical assays showing cross-reactivity of the antibodies to leiomodin- 1 and $O$. volvulus proteins; and (iv) that leiomodin-1 antibodies induce neurotoxicity. In combination, these data fulfill the second and third criteria for molecular mimicry. An epidemiological association between patients with nodding syndrome and $O$. volvulus infection previously fulfilled the first criterion for molecular mimicry. Further studies are needed to determine whether the autoantibodies can reproduce the clinical manifestations in an animal model. Such studies would provide direct evidence of an autoimmune process (32) and fulfill the fourth criterion for molecular mimicry.
The data presented here may have direct clinical translational value. Currently, patients with nodding syndrome are treated empirically with antiepileptic medications in an effort to control the seizures (33). Our data suggest that patients with nodding syndrome may benefit from immunomodulatory therapies, especially early in the course of disease before development of cognitive impairment. However, it is also possible that the immune responses may cause monophasic neurotoxicity, in which case immunotherapy may not be beneficial. Nevertheless, we provide evidence for specific CNS autoantibodies in patients with nodding syndrome, strengthen the association between nodding syndrome and $O$. volvulus infection, and suggest further areas of research that are critical for elucidating the pathobiology of nodding syndrome. 


\section{MATERIALS AND METHODS}

\section{Study design}

The overall objective of this work was to determine whether patients with nodding syndrome had autoantibodies that could contribute to disease pathogenesis. This was a cross-sectional study design conducted as a secondary analysis to the public health response activities initiated in Uganda and South Sudan. The comparisons made for discovery were between sera from patients with nodding syndrome $(n=19)$ and sera from unaffected village controls $(n=19)$. The sample size was based on the number of volunteers obtained within the collection period of 1 to 30 December 2009 from a single remote region in Northern Uganda affected in the epidemic. A second cohort of patients with nodding syndrome $(n=36)$ and unaffected village controls $(n=36)$ from South Sudan were included in the leiomodin-1 serum antibody analysis. Further comparisons between CSF from patients with nodding syndrome $(n=16)$ and U.S. patients with epilepsy $(n=8)$ were performed. Sample size for CSF was again based on the number of volunteers within the village. No CSF from unaffected village controls was obtained; therefore, U.S. patients were used as a control group. Investigators were blinded to the identity of the serum samples (patients or controls) during the initial screening for autoantibodies.

\section{Patient samples}

In response to an epidemic of nodding syndrome, the Ministry of Health in Uganda requested assistance with an investigation, which included the collection and testing of serum and CSF samples from patients with nodding syndrome, diagnosed using the consensus case definition (2), and serum samples from village controls unaffected by nodding syndrome or any other neurodevelopmental condition. Informed consent was obtained from all patients and controls. All samples were collected from 1 to 30 December 2009. Neuroimaging or electroencephalography was not performed on these persons at the time the samples were obtained because they lived in remote regions of Uganda. Onchocerca infection status was determined in this cohort by serology. Nineteen patients and 19 unaffected controls were matched for age within 5 years and were obtained from the same village. CSF was obtained from 16 patients with nodding syndrome. It was not considered appropriate to obtain CSF from unaffected village controls at that time. Deidentified CSF samples from eight U.S. patients with epilepsy and eight U.S. patients with pseudotumor cerebri were obtained by the National Institutes of Health (NIH) from the CSF repository at Johns Hopkins University, and their use for this study was approved by the NIH Office of Human Subjects Protection and Research. In South Sudan, serum samples from 36 patients with nodding syndrome and 36 unaffected village controls were obtained and screened for the presence of autoantibodies to leiomodin-1. Samples were collected between 26 May 2011 and 8 June 2011 by a joint team from the Ministry of Health, the Centers for Disease Control and Prevention (CDC), and the World Health Organization (WHO), with previous approval by the Ministry of Health in South Sudan. Informed consent was obtained from all patients and controls. In this cohort, Onchocerca infection status was determined by skin snip. Both of these public health investigations underwent regulatory review at the CDC and were determined to be nonresearch public health investigations.

\section{Unbiased screening for autoantibodies in sera}

To determine the diversity of autoantibodies in patients with nodding syndrome, we incubated two sets of pooled sera, one from patients with nodding syndrome and another from unaffected village controls on membranes spotted with $>9000$ full-length human proteins using an unbiased approach (that is, not restricted to known autoantigens) (Human ProtoArray v5.0; Invitrogen). The investigators were blinded to the identity of the pooled sera. Absolute differences in abundance of antibody recognition greater than twofold were further investigated, and the corresponding proteins were identified.

\section{Mass spectrometry}

Proteins were electrophoretically fractionated, and specific bands were excised and subjected to a modified in-gel digestion (34). Nanoflow reversed-phase liquid chromatography was performed using an Eksigent NanoLC-Ultra HPLC system (AB Sciex) directly coupled to a nanoelectrospray ionization ion source mounted on an LTQ Orbitrap Velos mass spectrometer (Thermo Fisher Scientific). MS1 spectra were acquired with a resolution of 30,000 at a mass/charge ratio of 400; MS2 spectra were acquired in the data-dependent mode. Tandem mass spectra were extracted from .RAW files using the MSConvert utility in the ProteoWizard Toolkit (version 3.0.3569) and output in .mgf format. Data sets were analyzed using the Mascot search engine (version 2.4.0; Matrix Science) against protein sequence libraries. The genomic data set of $O$. volvulus was downloaded from the Wellcome Trust Sanger Institute.

\section{Immunoblotting}

After approval by the Institutional Review Board at the NIH, adult human brain homogenate extracts (temporal cortex) from patients undergoing surgery for epilepsy, whole-organism lysates from $O$. volvulus, or overexpressed or recombinant proteins (leiomodin-1, OriGene; DJ-1, Abcam) were resolved by SDS-polyacrylamide gel electrophoresis and analyzed by immunoblotting pooled sera (1:5000), pooled CSF samples (1:1000), affinity-purified antibody (1:1000), or commercially available antibodies. Primary antibody incubations were performed overnight at $4^{\circ} \mathrm{C}$. Horseradish peroxidase (HRP) secondary antibodies (anti-human HRP or anti-rabbit HRP; 1:5000) were incubated at room temperature (RT) for 1 hour. Membranes were developed with enhanced chemiluminescence (Thermo Fisher Scientific). Blocking experiments were performed by incubating patient sera $(n=4)$ with either $10 \mu \mathrm{g}$ of BSA or $10 \mu \mathrm{g}$ of $O$. volvulus whole-organism lysates for 1 hour, followed by a 12-hour incubation with nitrocellulose-immobilized leiomodin-1. Immune subtyping was performed by immunoblotting leiomodin1 with patient sera (1:5000) and using chain-specific HRP-linked secondary antibodies. Blots were visualized with ProteinSimple FluorChem E/M Imager.

\section{Enzyme-linked immunosorbent assay}

High-binding plates were coated with purified recombinant leiomodin-1. Serum samples from patients and controls were diluted (1:200) and incubated overnight at $4^{\circ} \mathrm{C}$. Plates were incubated with anti-human HRP-coupled antibody for 1 hour at RT (1:5000). Plates were developed with $3,3^{\prime}, 5,5^{\prime}$-tetramethylbenzidine for $10 \mathrm{~min}$ at $\mathrm{RT}$, and development was stopped with $0.5 \mathrm{M}$ sulfuric acid. OD was read at $480 \mathrm{~nm}$ with a $560-\mathrm{nm}$ background correction on a FlexStation 3 (Molecular Devices). Positivity was defined by the mean \pm 3 SD from American healthy control blood donors $(n=20)$. Intraplate coefficient of variation $(\mathrm{CV})$ was $6 \%$, and interplate $\mathrm{CV}$ was $15.5 \%$. To ensure specificity of the assay, we used a subset of samples with the same protocol without antigen coupled. No signal was detected from these samples. 


\section{Immunoprecipitation}

Leiomodin-1 (accession number NP_036266; Life Technologies) (clone 6177902) was subcloned into the Pet16b expression vector (EMD Millipore), and in vitro transcription and translation (IVTT) was performed using the Promega T7 TNT expression system as per the manufacturer's instructions. One microliter of sera or CSF was incubated with $2 \mu \mathrm{l}$ of IVTT product for 1 hour at $4^{\circ} \mathrm{C}$. Antibodies were then captured with protein $\mathrm{G}$ beads and eluted from the beads by boiling in SDS buffer. Proteins were resolved by gel electrophoresis. Densities for each sample were calculated by the number of pixels per area after background correction. The cutoff for determining positive samples was mean intensity \pm 1 SD of readings obtained from American patients with pseudotumor cerebri (CSF; $n=8$ ). Samples from patients with pseudotumor cerebri were used to establish the assay but were not included in comparisons between groups of patients with epilepsy (U.S. patients versus patients with nodding syndrome).

\section{Overexpression of leiomodin-1}

HEK293T cells were plated in Dulbecco's modified Eagle's medium supplemented with $10 \%(\mathrm{v} / \mathrm{v})$ fetal bovine serum and $1 \%(\mathrm{v} / \mathrm{v})$ penicillinstreptomycin. Cells were transfected with either $0.4 \mu \mathrm{g}$ of leiomodin-1 using Lipofectamine 2000 as per the manufacturer's instructions (Life Technologies), empty vector control, or control pcDNA3.1-GFP (green fluorescent protein) vector to determine the transfection efficiency. Transfection efficiency was 90 to $95 \%$.

\section{Immunofluorescence}

Human neural progenitor cells were derived, cultured, and differentiated as previously described (35) after approval by the Office of $\mathrm{Hu}$ man Subjects Research and Protection at the NIH. Cells were fixed with $4 \%(\mathrm{w} / \mathrm{v})$ paraformaldehyde and immunostained with rabbit anti-leiomodin-1 (1:100) for 16 hours at $4^{\circ} \mathrm{C}$ and goat anti-rabbit Alexa Fluor 488 (Life Technologies, 1:400) for 2 hours at RT. Vybrant Dil cell-labeling solution (Invitrogen) was used to visualize the plasma membrane, and DAPI ( $1 \mu \mathrm{g} / \mathrm{ml}$; Roche Diagnostics) was used for nuclear staining (35). A Nikon Eclipse Ti total internal reflection fluorescence (TIRF) microscope equipped with a 488/561/405/647 LU4A Lasers System and an Andor iXon3 electron-multiplying chargecoupled device camera by using an Apo TIRF (100× objective; numerical aperture, 1.49) was used for imaging with acquisition (NISElements Advanced Research) and processing (ImageJ) to obtain the composite RGB images. For coimmunofluorescence with patient serum and CSF samples, cells were fixed, permeabilized with acetone, and incubated with rabbit anti-leiomodin-1 (1:100), patient sera (1:100), and patient CSF (1:25) for 1 hour at RT. Controls included secondary antibody-only staining (no primary antibody). After washing, cells were incubated with secondary antibodies conjugated to Alexa Fluor 594 (anti-rabbit) and Alexa Fluor 488 (anti-human) before incubation with DAPI. Cells were visualized on a laser scanning microscope (Zeiss LSM 510).

\section{Immunohistochemistry}

Immunohistochemistry for leiomodin-1 was performed on sagittal sections of adult mouse brain using leiomodin-1 polyclonal antibody (1:100; Proteintech). Murine smooth muscle from blood vessels and skeletal muscle were used as positive controls. Briefly, sections were deparaffinized and rehydrated with xylene substitute, followed by an ethanol gradient. Citrate buffer ( $\mathrm{pH}$ 6.0) was used for antigen retrieval. Primary antibody was incubated overnight at RT. PowerVision anti-rabbit HRP (Leica Biosystems) was applied for 2 hours at RT. Diaminobenzidine (Vector Laboratories) was used as a chromogen. Images were processed using a Nikon Eclipse Ci microscope. All murine tissues were collected according to the recommendations in the Guide for the Care and Use of Laboratory Animals of the NIH, and the use of these tissues was approved by the NIH.

\section{Quantitative PCR}

qPCR was performed on total RNA extracted using the RNeasy Plus Mini Kit (Qiagen) and treated with ribonuclease-free deoxyribonuclease from two independent adult human brain specimens, human neural stem cells, human neurons, HeLa cells, and H9C2 (myoblast) cells. RNA (375 ng) from each sample was reverse-transcribed into complementary DNA using SuperScript III (Invitrogen). Gene expression levels for leiomodin-1 and glyceraldehyde-3-phosphate dehydrogenase were acquired on an ABI PRISM 7000 Sequence Detection System (Applied Biosystems). All primers and standards were obtained from OriGene.

\section{Affinity purification of leiomodin-1 antibodies}

Antibodies to leiomodin-1 were affinity-purified from patient serum samples $(n=4)$. Purified recombinant leiomodin-1 was coupled to tosylactivated M280 beads as per the manufacturer's instructions (Life Technologies). Serum samples were incubated with leiomodin-1coupled beads for 1 hour at $37^{\circ} \mathrm{C}$. Sera depleted of leiomodin- 1 antibodies were used in neurotoxicity assays. Captured leiomodin-1 antibodies were eluted from the beads with $0.1 \mathrm{M}$ glycine $(\mathrm{pH} 2.0)$ and quickly returned to physiological $\mathrm{pH}$. Purified anti-leiomodin-1 antibodies were used in cross-reactivity studies.

\section{Neurotoxicity of leiomodin-1 antibodies}

Human primary neurons (iCell; Cellular Dynamics International) were cultured in laminin-coated (Sigma-Aldrich) 96 -well plates at $37^{\circ} \mathrm{C}[5 \%$ $(\mathrm{v} / \mathrm{v}) \mathrm{CO}_{2}$ ] and in iCell Complete Maintenance Medium (Cellular Dynamics International) at 80,000 cells per well. Before treatment, medium was replaced with Opti-MEM reduced-serum medium (Life Technologies). Neurons were treated with Opti-MEM only (negative control), rabbit IgG polyclonal anti-leiomodin-1 antibody $(20 \mu \mathrm{g} / \mathrm{ml})$, normal rabbit serum $(20 \mu \mathrm{g} / \mathrm{ml})$, patient sera $(n=1$, selected at random from patients with nodding syndrome; dilution of 1:200) or the same patient sera sample with all antibodies depleted $(n=1$; dilution of $1: 200)$, patient sera $(n=4$, selected at random from patients with nodding syndrome with detectable leiomodin-1 antibodies; dilution of 1:200), the same patient samples with leiomodin-1 antibodies depleted ( $n=4$; dilution of 1:200), or $0.1 \%(\mathrm{v} / \mathrm{v})$ saponin for 24 hours. Cell death was determined by uptake of propidium iodide (Cayman Chemical) as per the manufacturer's instructions. Neurons exposed to patient sera with and without antibody depletion or to CSF were tested for toxicity and viability using MultiTox-Fluor Multiplex Cytotoxicity Assay as per the manufacturer's instructions (Promega). Fluorescence was measured using FlexStation 3 Microplate Reader and SoftMax Pro software.

\section{Sequence homology analysis}

Sequences for human leiomodin-1 and O. volvulus were obtained from UniProt, and pairwise sequence alignment was performed using EMBOSS (EMBL-EBI). Local alignment was completed using the Smith-Waterman algorithm. Protein alignments and models were generated in ClustalW (version 12.0; Lasergene). 


\section{Statistics}

Results are presented as average OD for two replicates (ELISA), mean and SD of mean (qPCR), mORs with asymptotic 95\% CI (frequency of leiomodin-1 antibodies), or as percent of totals (viability and toxicity). Differences in OD of leiomodin-1 antibody binding in sera among cases and unaffected village controls were compared using ANOVA with Sidak-Holms correction for multiple comparisons. Frequency of leiomodin-1 antibodies in sera among cases and unaffected village controls was compared using an mOR test. Frequency of leiomodin-1 antibodies in CSF among cases and controls was compared using Fisher's exact test. Differences in neurotoxicity were assessed using two-way paired $t$ test. $P<0.05$ was considered statistically significant. All statistical analyses were performed with GraphPad Software 6.01 or SAS version 9.3.

\section{SUPPLEMENTARY MATERIALS}

www.sciencetranslationalmedicine.org/cgi/content/full/9/377/eaaf6953/DC1

Fig. S1. Representative image of immunoprecipitation experiments.

Fig. S2. Subtyping of leiomodin-1 autoantibodies from patients with nodding syndrome. Fig. S3. Immunofluorescence of leiomodin-1-transfected cells with sera from unaffected village controls.

Fig. S4. Coimmunofluorescence of human neurons with leiomodin-1 and patient CSF. Fig. S5. Leiomodin-1 transcripts are expressed in the brain as detected by RNA sequencing. Table S1. Proteins with enriched autoantibodies in patients with nodding syndrome. Table S2. Top four proteins with enriched autoantibodies in patients with nodding syndrome. Table S3. Leiomodin-1 transcripts are expressed in the brain.

Table S4. Rabbit leiomodin-1 antibodies are neurotoxic.

Table S5. Antibodies in patient sera are neurotoxic.

Table S6. Leiomodin-1 antibodies from patients are neurotoxic.

Table S7. O. volvulus proteins identified by mass spectrometry.

Table S8. Leiomodin-1 antibodies cross-react with $O$. volvulus antigens.

\section{REFERENCES AND NOTES}

1. J. J. Sejvar, A. M. Kakooza, J. L. Foltz, I. Makumbi, A. D. Atai-Omoruto, M. Malimbo, R. Ndyomugyenyi, L. N. Alexander, B. Abang, R. G. Downing, A. Ehrenberg, K. Guilliams, S. Helmers, P. Melstrom, D. Olara, S. Perlman, J. Ratto, E. Trevathan, A. S. Winkler, S. F. Dowell, D. Lwamafa, Clinical, neurological, and electrophysiological features of nodding syndrome in Kitgum, Uganda: An observational case series. Lancet Neurol. 12, 166-174 (2013).

2. S. F. Dowell, J. J. Sejvar, L. Riek, K. A. H. Vandemaele, M. Lamunu, A. C. Kuesel, E. Schmutzhard, W. Matuja, S. Bunga, J. Foltz, T. B. Nutman, A. S. Winkler, A. K. Mbonye, Nodding syndrome. Emerg. Infect. Dis. 19, 1374-1384 (2013).

3. R. Idro, R. O. Opoka, H. T. Aanyu, A. Kakooza-Mwesige, T. Piloya-Were, H. Namusoke, S. B. Musoke, J. Nalugya, P. Bangirana, A. D. Mwaka, S. White, K. Chong, A. D. Atai-Omoruto, E. Mworozi, J. Nankunda, S. Kiguli, J. R. Aceng, J. K. Tumwine, Nodding syndrome in Ugandan children-Clinical features, brain imaging and complications: A case series. BMJ Open 3, 10.1136/bmjopen-2012-002540 (2013).

4. A. S. Winkler, K. Friedrich, R. König, M. Meindl, R. Helbok, I. Unterberger, T. Gotwald, J. Dharsee, S. Velicheti, A. Kidunda, L. Jilek-Aall, W. Matuja, E. Schmutzhard, The head nodding syndrome-Clinical classification and possible causes. Epilepsia 49, 2008-2015 (2008).

5. A. S. Winkler, K. Friedrich, S. Velicheti, J. Dharsee, R. König, A. Nassri, M. Meindl, A. Kidunda, T. H. Müller, L. Jilek-Aall, W. Matuja, T. Gotwald, E. Schmutzhard, MRI findings in people with epilepsy and nodding syndrome in an area endemic for onchocerciasis: An observational study. Afr. Health Sci. 13, 529-540 (2013).

6. J. L. Foltz, I. Makumbi, J. J. Sejvar, M. Malimbo, R. Ndyomugyenyi, A. D. Atai-Omoruto, L. N. Alexander, B. Abang, P. Melstrom, A. M. Kakooza, D. Olara, R. G. Downing, T. B. Nutman, S. F. Dowell, D. K. W. Lwamafa, An epidemiologic investigation of potential risk factors for nodding syndrome in Kitgum district, Uganda. PLOS ONE 8, e66419 (2013).

7. R. König, A. Nassri, M. Meindl, W. Matuja, A. R. Kidunda, V. Siegmund, G. Bretzel, T. Löscher, L. Jilek-Aall, E. Schmutzhard, A. S. Winkler, The role of Onchocerca volvulus in the development of epilepsy in a rural area of Tanzania. Parasitology 137, 1559-1568 (2010).
8. B. O. Duke, J. Vincelette, P. J. Moore, Microfilariae in the cerebrospinal fluid, and neurological complications, during treatment of onchocerciasis with diethylcarbamazine. Tropenmed. Parasitol. 27, 123-132 (1976).

9. A. Dietmann, B. Wallner, R. König, K. Friedrich, B. Pfausler, F. Deisenhammer, A. Griesmacher, C. Seger, W. Matuja, L. JilekAall, A. S. Winkler, E. Schmutzhard, Nodding syndrome in Tanzania may not be associated with circulating anti-NMDA-and anti-VGKC receptor antibodies or decreased pyridoxal phosphate serum levels-a pilot study. Afr. Health Sci. 14, 434-438 (2014).

10. C. A. Conley, K. L. Fritz-Six, A. Almenar-Queralt, V. M. Fowler, Leiomodins: Larger members of the tropomodulin (Tmod) gene family. Genomics 73, 127-139 (2001).

11. V. Nanda, J. M. Miano, Leiomodin 1, a new serum response factor-dependent target gene expressed preferentially in differentiated smooth muscle cells. J. Biol. Chem. 287, 2459-2467 (2012).

12. S. C. P. Williams, Nodding syndrome leaves baffled scientists shaking their heads. Nat. Med. 18, 334 (2012).

13. J. K. Tumwine, K. Vandemaele, S. Chungong, M. Richer, M. Anker, Y. Ayana, M. L. Opoka, D. N. Klaucke, A. Quarello, P. S. Spencer, Clinical and epidemiologic characteristics of nodding syndrome in Mundri County, southern Sudan. Afr. Health Sci. 12, 242-248 (2012).

14. P. S. Spencer, K. Vandemaele, M. Richer, V. S. Palmer, S. Chungong, M. Anker, Y. Ayana M. L. Opoka, B. N. Klaucke, A. Quarello, J. K. Tumwine, Nodding syndrome in Mundri county, South Sudan: Environmental, nutritional and infectious factors. Afr. Health Sci. 13, 183-204 (2013).

15. H. C. Santiago, S. Bennuru, A. Boyd, M. Eberhard, T. B. Nutman, Structural and immunologic cross-reactivity among filarial and mite tropomyosin: Implications for the hygiene hypothesis. J. Allergy Clin. Immunol. 127, 479-486 (2011).

16. P. J. lyengar, J. Wamala, J. Ratto, C. Blanton, M. Malimbo, L. Lukwago, S. Becknell, R. Downing, S. Bunga, J. Sejvar, I. Makumbi, Prevalence of nodding syndrome-Uganda, 2012-2013. MMWR Morb. Mortal. Wkly Rep. 63, 603-606 (2014).

17. A. S. Winkler, K. Friedrich, M. Meindl, A. Kidunda, A. Nassri, L. Jilek-Aall, W. Matuja, E. Schmutzhard, Clinical characteristics of people with head nodding in southern Tanzania. Trop. Doct. 40, 173-175 (2010).

18. X. Qiao, T. R. Werkman, J. A. Gorter, W. J. Wadman, E. A. van Vliet, Expression of sodium channel $\alpha$ subunits $1.1,1.2$ and 1.6 in rat hippocampus after kainic acid-induced epilepsy. Epilepsy Res. 106, 17-28 (2013).

19. Q. Dong, M. Ludgate, G. Vassart, Cloning and sequencing of a novel 64-kDa autoantigen recognized by patients with autoimmune thyroid disease. J. Clin. Endocrinol. Metab. 72 1375-1381 (1991).

20. D. Chereau, M. Boczkowska, A. Skwarek-Maruszewska, I. Fujiwara, D. B. Hayes, G. Rebowski, P. Lappalainen, T. D. Pollard, R. Dominguez, Leiomodin is an actin filament nucleator in muscle cells. Science 320, 239-243 (2008).

21. S. Wright, A. Vincent, Progress in autoimmune epileptic encephalitis. Curr. Opin. Neurol. 29, 151-157 (2016)

22. C. Geis, A. Weishaupt, S. Hallermann, B. Grünewald, C. Wessig, T. Wultsch, A. Reif, N. Byts, M. Beck, S. Jablonka, M. K. Boettger, N. Üçeyler, W. Fouquet, M. Gerlach, H.-M. Meinck, A.-L. Sirén, S. J. Sigrist, K. V. Toyka, M. Heckmann, C. Sommer, Stiff person syndromeassociated autoantibodies to amphiphysin mediate reduced GABAergic inhibition. Brain 133, 3166-3180 (2010).

23. J. C. Hall, L. Casciola-Rosen, A. Rosen, Altered structure of autoantigens during apoptosis. Rheum. Dis. Clin. North Am. 30, 455-471, vii (2004).

24. L. Casciola-Rosen, A. Rosen, M. Petri, M. Schlissel, Surface blebs on apoptotic cells are sites of enhanced procoagulant activity: Implications for coagulation events and antigenic spread in systemic lupus erythematosus. Proc. Natl. Acad. Sci. U.S.A. 93, 1624-1629 (1996).

25. L. Casciola-Rosen, K. Nagaraju, P. Plotz, K. Wang, S. Levine, E. Gabrielson, A. Corse, A. Rosen, Enhanced autoantigen expression in regenerating muscle cells in idiopathic inflammatory myopathy. J. Exp. Med. 201, 591-601 (2005).

26. R. Idro, B. Opar, J. Wamala, C. Abbo, S. Onzivua, D. A. Mwaka, A. Kakooza-Mwesige, A. Mbonye, J. R. Aceng, Is nodding syndrome an Onchocerca volvulus-induced neuroinflammatory disorder? Uganda's story of research in understanding the disease. Int. J. Infect. Dis. 45, 112-117 (2016).

27. T. Piloya-Were, B. Odongkara-Mpora, H. Namusoke, R. Idro, Physical growth, puberty and hormones in adolescents with Nodding Syndrome; a pilot study. BMC. Res. Notes 7, 858 (2014),

28. J. F. Wamala, M. Malimbo, F. Tepage, L. Lukwago, C. L. Okot, R. O. Cannon, A. Laudisoit, R. Colebunders, Nodding syndrome may be only the ears of the Hippo. PLOS Negl. Trop. Dis. 9, e0003880 (2015).

29. R. Höftberger, M. Sepulveda, T. Armangue, Y. Blanco, K. Rostásy, A. Cobo Calvo, J. Olascoaga, L. Ramió-Torrentà, M. Reindl, J. Benito-León, B. Casanova, G. Arrambide, L. Sabater, F. Graus, J. Dalmau, A. Saiz, Antibodies to MOG and AQP4 in adults with neuromyelitis optica and suspected limited forms of the disease. Mult. Scler. 21, 866-874 (2014). 30. M. Petit-Pedrol, T. Armangue, X. Peng, L. Bataller, T. Cellucci, R. Davis, L. McCracken, E. Martinez-Hernandez, W. P. Mason, M. C. Kruer, D. G. Ritacco, W. Grisold, B. F. Meaney, 
C. Alcalá, P. Sillevis-Smitt, M. J. Titulaer, R. Balice-Gordon, F. Graus, J. Dalmau, Encephalitis with refractory seizures, status epilepticus, and antibodies to the GABAA receptor: A case series, characterisation of the antigen, and analysis of the effects of antibodies. Lancet Neurol. 13, 276-286 (2014)

31. C. W. Ang, B. C. Jacobs, J. D. Laman, The Guillain-Barré syndrome: A true case of molecular mimicry. Trends Immunol. 25, 61-66 (2004).

32. N. R. Rose, C. Bona, Defining criteria for autoimmune diseases (Witebsky's postulates revisited). Immunol. Today 14, 426-430 (1993).

33. R. Idro, H. Namusoke, C. Abbo, B. B. Mutamba, A. Kakooza-Mwesige, R. O. Opoka, A. K. Musubire, A. D. Mwaka, B. T. Opar, Patients with nodding syndrome in Uganda improve with symptomatic treatment: A cross-sectional study. BMJ Open 4, e006476 (2014).

34. P. T. Matsudaira, A Practical Guide to Protein and Peptide Purification for Microsequencing (Academic Press, ed. 2, 1993), $184 \mathrm{pp.}$

35. T. Wang, E. Choi, M. C. G. Monaco, E. Campanac, M. Medynets, T. Do, P. Rao, K. R. Johnson, A. G. Elkahloun, G. Von Geldern, T. Johnson, S. Subramaniam, D. Hoffman, E. Major, A. Nath, Derivation of neural stem cells from human adult peripheral CD34+ cells for an autologous model of neuroinflammation. PLOS ONE 8, e81720 (2013).

Acknowledgments: We thank B. E. Johnson for statistical support, C. Pardo for providing U.S. CSF samples, M. Konig for critically reading the manuscript, M. C. Haffner for assistance in manuscript preparation and useful discussion, the Rosen Laboratory at Johns Hopkins University for helpful comments, and partners for the public health response in the affected communities from Uganda and South Sudan. Funding: This research was supported by the Intramural Research Programs of the NIH and CDC. The findings and conclusions of this report are those of the authors and do not necessarily represent the official position of the CDC. Author contributions: T.P.J. designed and conducted most of the experiments, generated the hypotheses, analyzed the data, and wrote the manuscript. R.T. conducted gene expression experiments. P.R.L. performed neurotoxicity experiments. M.-H.L. performed immunohistochemistry. K.R.J. analyzed the protein array data and sequence homology data. J.K. conducted the mass spectrometry experiments. A.E. performed the protein array experiment. M.M. developed the in vitro cultures of neurons and neural progenitor cells and associated immunostaining. A.H. performed the TIRF studies. J.K. assayed for anti-Onchocerca antibodies. J.R. and S.B. managed and analyzed patient data. J.S., S.F.D., I.M., and J.R.A. characterized the patients and controls, collected the samples, generated the hypotheses, and assisted in writing the manuscript. T.B.N. analyzed the data, generated the hypotheses, performed three-dimensional protein modeling, and assisted in writing the manuscript. A.N. supervised the project, generated the hypotheses, designed the experiments, analyzed the data, and helped in writing the manuscript. Competing interests: The authors declare that they have no competing interests.

Submitted 16 March 2016

Accepted 17 November 2016

Published 15 February 2017

10.1126/scitransImed.aaf6953

Citation: T. P. Johnson, R. Tyagi, P. R. Lee, M.-H. Lee, K. R. Johnson, J. Kowalak, A. Elkahloun, M. Medynets, A. Hategan, J. Kubofcik, J. Sejvar, J. Ratto, S. Bunga, I. Makumbi, J. R. Aceng, T. B. Nutman, S. F. Dowell, A. Nath, Nodding syndrome may be an autoimmune reaction to the parasitic worm Onchocerca volvulus. Sci. Transl. Med. 9, eaaf6953 (2017). 
Science

Translational

Medicine

MAAAS
Nodding syndrome may be an autoimmune reaction to the parasitic worm Onchocerca volvulus

Tory P. Johnson, Richa Tyagi, Paul R. Lee, Myoung-Hwa Lee, Kory R. Johnson, Jeffrey Kowalak, Abdel Elkahloun, Marie Medynets, Alina Hategan, Joseph Kubofcik, James Sejvar, Jeffrey Ratto, Sudhir Bunga, Issa Makumbi, Jane R. Aceng, Thomas B. Nutman, Scott F. Dowell and Avindra Nath (February 15, 2017) Science Translational Medicine 9 (377), . [doi:

10.1126/scitranslmed.aaf6953]

Editor's Summary

\section{Linking parasitic infection to autoimmune epilepsy}

Nodding syndrome is a unique seizure disorder affecting children in parts of East Africa. The cause of nodding syndrome has been an enigma, although an epidemiological association with the parasite Onchocerca volvulus has been established. Johnson et al. demonstrate that patients with nodding syndrome have autoantibodies to leiomodin-1 that are neurotoxic in vitro and that leiomodin-1 is expressed in regions of the brain affected during disease. Leiomodin- 1 antibodies cross-react with $O$. volvulus proteins, linking the parasite to the autoantibody. Thus, nodding syndrome may be an autoimmune epilepsy initiated by a parasitic infection and may be preventable by treatment with antiparasitic strategies such as the drug ivermectin.

The following resources related to this article are available online at http://stm.sciencemag.org. This information is current as of March 9, 2017.

Article Tools Visit the online version of this article to access the personalization and article tools:

http://stm.sciencemag.org/content/9/377/eaaf6953

Supplemental Materials

Related Content

Permissions
"Supplementary Materials"

http://stm.sciencemag.org/content/supp1/2017/02/13/9.377.eaaf6953.DC1

The editors suggest related resources on Science's sites:

http://stm.sciencemag.org/content/scitransmed/9/377/eaam8532.full http://science.sciencemag.org/content/sci/355/6326/678.full

Obtain information about reproducing this article: http://www.sciencemag.org/about/permissions.dtl

Science Translational Medicine (print ISSN 1946-6234; online ISSN 1946-6242) is published weekly, except the last week in December, by the American Association for the Advancement of Science, 1200 New York Avenue, NW, Washington, DC 20005. Copyright 2017 by the American Association for the Advancement of Science; all rights reserved. The title Science Translational Medicine is a registered trademark of AAAS. 